\title{
ATUAÇÃO DO FISIOTERAPEUTA NO ATENDIMENTO A PACIENTES COM FIBROSE CÍSTICA: UMA REVISÃO DE LITERATURA
}

\footnotetext{
Laíza Gonçalves Silva1, Yasmim Queiroz Santos², Lucieli Boschetti Vinhal³ Flávio Monteiro Ayres

1. Graduanda do curso de Fisioterapia da Universidade Estadual de Goiás, Goiânia - Brasil laizagsilva22@gmail.com

2. Bacharel em Fisioterapia e discente no Programa de Mestrado em Ciências Aplicadas a Produtos para a Saúde pela Universidade Estadual de Goiás, Goiânia

3. Mestre em Terapia Intensiva pela Sociedade Brasileira de Terapia Intensiva e Especialista em Fisioterapia Intensiva e Respiratória

4. Doutor em Controle de Funções Biológicas e docente dos cursos Fisioterapia, Mestrado em Ciências Moleculares e Mestrado em Ciências Aplicadas a Produtos para a Saúde da Universidade Estadual de Goiás

Recebido em: 08/04/2017 - Aprovado em: 10/06/2017 - Publicado em: 20/06/2017 DOI: 10.18677/EnciBio_2017A123
}

\section{RESUMO}

A fibrose cística é uma doença autossômica recessiva, pleiotrópica e deletéria, que compromete o funcionamento dos sistemas digestivo, musculoesquelético e respiratório. Essa revisão de literatura abordou a atuação do fisioterapeuta no atendimento a pacientes com fibrose cística mediante dados bibliográficos disponíveis no PubMed, SciELO e PEDro entre os anos 2006 a 2016. Os resultados encontrados evidenciam que a fisioterapia tem um papel importante no tratamento do paciente com fibrose cística, promovendo principalmente a melhora da qualidade de vida e aumentado também a sobrevida. A atuação do fisioterapeuta no atendimento desses pacientes contempla: 1) fisioterapia respiratória, que auxilia na eliminação de secreções das vias aéreas em pacientes com fibrose cística utilizando técnicas clássicas e atuais como, drenagem postural, vibração e percussão, técnica de expiração forçada, técnica do ciclo ativo da respiração, pressão expiratória positiva oscilatória - flutter, pressão expiratória positiva e drenagem autógena; 2) fisioterapia nas alterações musculoesqueléticas, que inclui exercícios de cinesioterapia com resistência para a melhora da força muscular do paciente; 3) terapia manual visceral, que estimula e aumenta o peristaltismo, auxilia a secreção de fluidos glandulares, reduz o tempo de trânsito colônico e diminui a dor e o desconforto causados pelas disfunções digestivas; 4) 0 envolvimento e comprometimento da família, que aumenta a adesão do paciente ao tratamento e melhora efetivamente a avaliação prognóstica. Em conclusão, o tratamento fisioterapêutico da fibrose cística proporciona a melhora global do quadro fisiopatológico destes pacientes.

PALAVRAS-CHAVE: CFTR, mucoviscosidade e técnicas fisioterapêuticas 


\title{
PHYSIOTHERAPIST PERFORMANCE IN THE CARE OF PATIENTS WITH CYSTIC FIBROSIS: A LITERATURE REVIEW
}

\begin{abstract}
Cystic fibrosis is an autosomal recessive, pleiotropic and deleterious disease that compromises the functioning of the digestive, musculoskeletal, and respiratory systems. This literature review addressed the physiotherapist's performance not attending patients with cystic fibrosis through bibliographic data available in PubMed, SciELO and PEDRO between the years 2006 to 2016. The results found evidence that physiotherapy has an important role not to treat the patient with Cystic fibrosis, mainly promoting an improvement in the quality of life and also increased survival. The physiotherapist's role in the care of these patients includes: 1) respiratory physiotherapy, which assists in the elimination of airway secretions in patients with cystic fibrosis using classical and current techniques such as postural drainage, vibration and percussion, forced expiration technique, Active breathing cycle, oscillatory positive expiratory pressure - flutter, positive expiratory pressure and autogenous drainage; 2) physiotherapy in musculoskeletal disorders, which includes kinesiotherapy exercises with resistance to improve the patient's muscular strength; 3) visceral manual therapy, which stimulates and increases peristalsis, aids the secretion of glandular fluids, reduces colonic transit time, and decreases pain and discomfort caused by digestive dysfunctions; 4) family involvement and commitment, which increases patient adherence to treatment and effectively improves prognostic evaluation. In conclusion, the physiotherapeutic treatment of cystic fibrosis provides the overall improvement of the pathophysiological picture of these patients.
\end{abstract}

KEYWORDS: CFTR, mucoviscosity and physiotherapeutic techniques

\section{INTRODUÇÃO}

A fibrose cística, conhecida também como mucoviscidose, é uma doença genética, de caráter autossômico recessivo, crônica e progressiva que compromete principalmente $\mathrm{o}$ funcionamento dos sistemas digestivo, respiratório e musculoesquelético (ZIEGLER et al., 2009; AGUIAR et al., 2016). Aproximadamente, 2.000 mutações referentes a fibrose cística foram descobertas. Essas mutações ocorrem no gene localizado no braço longo do cromossomo 7, posição q31. O gene cttr é encarregado de codificar uma proteína de 1.480 aminoácidos, a cystic fibrosis transmembrane condutance regulator (DALCIN \& SILVA, 2008; COUTINHO et al., 2013; QUON \& ROWE, 2016).

A deficiência de CFTR tem como consequência uma alteração da viscosidade de secreções, resultando em uma doença pulmonar progressiva. O indivíduo poderá manifestar também disfunções no sistema digestivo, musculoesquelético e altos níveis de eletrólitos no suor (COHEN et al., 2011). Dentre as manifestações clínicas pulmonares mais habituais, tem-se bronquite, sinusite, bronquiectasia e pneumonia. Devido à falta de enzimas liberadas do pâncreas para o duodeno, irá ocorrer uma má absorção de proteínas, carboidratos e gorduras, causando diarreia crônica e desnutrição. Pacientes com fibrose cística apresentam também redução da força muscular, contribuindo para a fadiga durante a prática de exercício e atividades diárias (SUNDELL et al., 2012; FILHO et al., 2013).

A fibrose cística é uma doença pleiotrópica, ou seja, apresenta características fenotípicas diversas. Resultando, assim, em diferenças clínicas significativas no grau de severidade da doença e nas suas complicações para cada paciente (ZIEGLER et 
al., 2009; TORRES et al., 2010). Tendo em vista essas variedades dos sintomas, cada paciente passa por uma avaliação individual e posteriormente será traçado um tratamento específico. Nesse sentido, o tratamento ressalta as maiores incapacidades do paciente e, dessa forma não há um padrão para o tratamento da fibrose cística (GOMIDE et al., 2007; CONTO et al., 2014).

O tratamento para a fibrose cística deve ser realizado e acompanhado por uma equipe multidisciplinar, composta por médico, nutricionista, fisioterapeuta, enfermeiro, assistente social e psicólogo, dentre outros. A fisioterapia, há muito tempo, tem representado um papel importante no tratamento da fibrose cística, promovendo principalmente a melhora da qualidade de vida e aumentado também a sobrevida do paciente fibrocístico. Dessa forma o indivíduo consegue realizar atividades de vida diária o mais próximo da normalidade (PRADO, 2011; FEITEN et al., 2016; DONADIO et al., 2013). O objetivo desta revisão de literatura foi abordar a atuação do fisioterapeuta no atendimento a pacientes com fibrose cística.

A pesquisa da literatura foi realizada nas bases de dados bibliográficas e eletrônicas, PubMed, SciELO e PEDro no período de 2006 a 2016. As palavraschave usadas em várias combinações foram: "fibrose cística", "fisioterapia na fibrose cística" e "técnicas fisioterápicas", "cystic fibrosis", "physiotherapy in cystic fibrosis", "physiotherapeutic techniques", "fibrosis quística", "fisioterapia en la fibrosis quística", fisioterapia técnica". A pesquisa abrangeu artigos de língua portuguesa, inglesa e espanhol que foram publicados no referido período.

\section{Avaliação fisioterapêutica}

Os instrumentos de avaliação para verificar a progressão da doença e a intervenção no tratamento incluem análise da gasometria arterial, os testes de função pulmonar que fornecem o percentual do VEF1 (volume expiratório final no primeiro segundo) e a oximetria que analisa a $\mathrm{SpO} 2$ (saturação periférica de oxigênio). O fisioterapeuta deverá se atentar para outras ferramentas importantes de avaliação como, a cultura de escarro, ausculta pulmonar, capacidade física do paciente, verificação qualitativa da dispneia e dor, peso corporal e IMC e exames de imagem (TORRES et al., 2010; PRADO 2011; HEINZMANN-FILHO et al., 2015).

Após a realização do diagnóstico da doença, deve ser feita uma avaliação fisioterapêutica individualizada, obtendo informações sobre a histórica médica do paciente, o estado clínico atual da doença, avaliando sinais e sintomas e os fatores desencadeadores e tratamentos realizados anteriormente (CASTRO \& FIRMIDA, 2011). O exame físico fornecerá informações essenciais para a prescrição do tratamento, onde serão observados os sinais clínicos como, uso excessivo da musculatura respiratória, tipo e mobilidade do tórax, presença de cianose e os padrões ventilatórios. Posteriormente, estes achados deverão ser correlacionados aos exames de imagem e a ausculta pulmonar (DALCIN \& SILVA, 2008; CARDOSO \& VIANA, 2011).

A ausculta pulmonar servirá ao fisioterapeuta de orientação para traçar o tratamento e as técnicas adequadas devido a presença dos sons normais ou anormais por todo o pulmão, detectará também as áreas de hipoventilação ou não ventiladas com acúmulo de secreções ou outros sinais clínicos (PRADO, 2011).

As alterações musculoesqueléticas são frequentes em pacientes com fibrose cística como, a redução da força muscular que irá contribuir para a fadiga durante as atividades diária e a prática de exercício. Em vista disso, o fisioterapeuta deverá realizar uma avaliação minuciosa e individual para ressaltar as maiores 
incapacidades e assim traçar um tratamento específico e individualizado (GOMIDE et al., 2007; CONTO et al., 2014).

\section{Técnicas de Fisioterapia Respiratória}

A fisioterapia respiratória tem representado um papel importante na eliminação de secreções das vias aéreas em pacientes com fibrose cística e geralmente se inicia logo após a confirmação do diagnóstico. Deve-se estabelecer um programa de tratamento específico de acordo com o estado clínico atual de cada paciente. Esse tratamento deverá ser feito diariamente e alterado conforme a progressão da doença (SILVA \& PFEIFER, 2007; CARDOSO \& VIANA, 2011).

As técnicas de higiene brônquica auxiliam na eliminação de secreções diminuindo a obstrução das vias aéreas e suas complicações (quadro 1). As manobras clássicas são drenagem postural, vibração e percussão (CASTAÑOS \& RENTERÍA, 2008). Outras alternativas mais recentes incluem, técnica de expiração forçada (TEF), técnica do ciclo ativo da respiração (CAR), pressão expiratória positiva (PEP) e drenagem autógena (WARNOCK \& GATES, 2015).

QUADRO 1: Manobras respiratórias e recursos terapêuticos utilizados em pacientes com fibrose cística

\begin{tabular}{|c|c|c|}
\hline Técnica & Prática & Objetivos \\
\hline $\begin{array}{l}\text { Drenagem } \\
\text { Postural }\end{array}$ & $\begin{array}{l}\text { Posições que utilizam a ação } \\
\text { da gravidade, tempo de } \\
\text { permanência de } 3 \text { a } 15 \\
\text { minutos }\end{array}$ & $\begin{array}{l}\text { Mobilizar as secreções das } \\
\text { vias aéreas periféricas } \\
\text { para as vias aéreas } \\
\text { centrais }\end{array}$ \\
\hline Percussão & $\begin{array}{l}\text { Manobras realizadas no tórax } \\
\text { gerando vibrações } \\
\text { intrapulmonares }\end{array}$ & $\begin{array}{l}\text { Mobilizar as secreções das } \\
\text { paredes pulmonares, } \\
\text { estimulando a tosse }\end{array}$ \\
\hline Vibração & $\begin{array}{l}\text { Movimentos rítmicos } \\
\text { oscilatórios realizados no } \\
\text { tórax, somente na expiração }\end{array}$ & $\begin{array}{l}\text { Aumenta o fluxo expiratório } \\
\text { e desloca as secreções }\end{array}$ \\
\hline TEF & $\begin{array}{l}\text { Uma ou duas expirações } \\
\text { forçadas com a glote aberta, } \\
\text { seguida de tosse }\end{array}$ & $\begin{array}{l}\text { Mobilizar e expectorar as } \\
\text { secreções }\end{array}$ \\
\hline CAR & $\begin{array}{l}\text { Técnica ativa do controle } \\
\text { respiratório seguida de TEF }\end{array}$ & $\begin{array}{l}\text { Impede a dessaturação do } \\
\text { oxigênio e auxilia na } \\
\text { expectoração }\end{array}$ \\
\hline PEP & $\begin{array}{l}\text { Máscara facial que possui uma } \\
\text { resistência na fase expiratória }\end{array}$ & $\begin{array}{l}\text { Reduzir o } \text { aprisionamento } \\
\text { de ar a mobilizar } \\
\text { secreções }\end{array}$ \\
\hline Flutter & $\begin{array}{l}\text { Aparelho que combina as } \\
\text { técnicas PEP com oscilações } \\
\text { de alta frequência }\end{array}$ & $\begin{array}{l}\text { Melhora os mecanismos } \\
\text { de eliminação da secreção }\end{array}$ \\
\hline $\begin{array}{l}\text { Drenagem } \\
\text { Autógena }\end{array}$ & Utiliza o alto fluxo expiratório & $\begin{array}{l}\text { Mobilizar secreções das } \\
\text { vias aéreas distais para } \\
\text { centrais }\end{array}$ \\
\hline
\end{tabular}

TEF: Técnica de Expiração Forçada; CAR: Técnica do Ciclo Ativo da Respiração; PEP: Pressão Expiratória Positiva 


\section{Drenagem Postural (DP)}

A drenagem postural é descrita como o uso de posições que se utilizam da ação da gravidade para ajudar na mobilização das secreções no trato respiratório, mobilizando as secreções das vias aéreas periféricas para as vias aéreas centrais, onde poderão ser removidas por meio da tosse. O tempo recomendado da DP é entre 3 e 15 minutos por posição escolhida, sendo que o tempo total não exceda 30 a 40 minutos. As contraindicações para essa técnica são: pressão intracraniana elevada, lesão medular aguda, cardiopatias agudas e crônicas, derrames pleurais, instabilidade hemodinâmica, traumatismo torácico e insuficiência respiratória (IKE et al., 2009; SILVA \& BROMERSCHENCKEL, 2013).

Para obter efeitos ainda melhores, a drenagem postural associada a técnica de expiração forçada é capaz de potencializar a função pulmonar pelo aumento da capacidade funcional forçada (CVF), do pico de fluxo expiratório (PFE) e do VEF1. Outras manobras que podem ser associadas a drenagem postural são a percussão e vibração com objetivo de auxiliar na desobstrução das vias aéreas. A drenagem postural é considerada uma técnica necessária para higiene brônquica em pacientes com fibrose cística. No entanto, é valido lembrar que, para se apresentar mais eficaz, essa técnica deve estar ligada a outras manobras e à correta hidratação das vias aéreas (GOMIDE et al., 2007; IKE et al., 2009).

\section{Percussão e Vibração Manual}

A percussão é um conjunto de manobras realizadas na superfície externa do tórax gerando vibrações intrapulmonares que irá deslocar e mobilizar as secreções na direção da traqueia, estimulando a tosse e a eliminação da secreção. Pode ser realizada de três formas: punho percussão ou percussão cubital e tapotagem. $O$ tempo deve ser definido de acordo com as condições clínicas e individuais de cada paciente, permanecendo entre 3 a 5 minutos para cada área do tórax que apresenta acúmulo de secreção. As contraindicações para essa técnica são, edema agudo de pulmão, fratura de costelas, metástase do pulmão ou mediastino, crise asmática, arritmias cardíacas importantes e osteoporose grave (LIEBANO et al., 2009; SILVA \& BROMERSCHENCKEL, 2013).

A vibração manual inclui movimentos rítmicos oscilatórios realizados na parede torácica do paciente durante a fase expiratória para aumentar o fluxo expiratório, e obter o deslocamento das secreções. Esses movimentos podem ser associados com a compressão da caixa torácica afim de potencializar o aumento do fluxo expiratório. A intensidade e a força utilizada nessa manobra são ajustadas às necessidades individuais do paciente (CASTAÑOS \& RENTERÍA, 2008; PRADO, 2011; OLIVEIRA \& GOMES, 2016).

\section{Técnica de Expiração Forçada (TEF)}

A técnica de expiração forçada pode ser chamada também de huffing, consiste em uma ou duas expirações forçadas, com a glote aberta, seguida de tosse para expectoração da secreção e um período de respiração diafragmática controlada. Esse período é importante para evitar queda da saturação e broncoespasmos. O huff de volume pulmonar baixo moverá secreções periféricas, enquanto um huff de alto volume pulmonar removerá a mucosidade localizada nas partes proximais das vias aéreas superiores (SILVA \& BROMERSCHENCKEL, 2013).

O objetivo dessa manobra é favorecer a remoção de secreções brônquicas ENCICLOPÉDIA BIOSFERA, Centro Científico Conhecer - Goiânia, v.14 n.25; p. 1479 2017 
aglomeradas com a menor alteração de pressão pleural. A técnica de expiração tem sido bastante eficaz na higiene brônquica de pacientes que tendem a ter o colapso das vias aéreas durante a tosse normal, como é o caso dos pacientes com fibrose cística. A TEF associada a drenagem postural mostrou-se bastante eficaz para o aumento da quantidade de secreção expectorada e no aumento da função pulmonar, gerando um aumento considerável na VEF1 e na capacidade funcional forçada (GOMIDE et al., 2007; WARNOCK \& GATES, 2015).

\section{Técnica do Ciclo Ativo da Respiração (CAR)}

O ciclo ativo da respiração é dividido em 3 etapas: controle da respiração, expansão torácica e técnica de expansão torácica. A primeira etapa implica o controle respiratório por meio de respirações predominantemente diafragmáticas, visando a precaução de broncoespasmo. A segunda etapa envolve exercícios de expansão pulmonar que inclui inspirações lentas e profundas com expirações tranquilas. Essa etapa deve conter de 6 a 8 exercícios para remover a secreção e aprimorar a ventilação. A última e terceira etapa consistem em executar a técnica de expiração forçada para mover as secreções para vias aéreas superiores (FLUME et al., 2009; PRADO, 2011). O ciclo ativo da respiração associado a drenagem postural é bastante eficaz por reduzir a chances de dessaturação de oxigênio que normalmente ocorre durante a terapia de drenagem postural em pacientes com fibrose cística, otimizando também o desempenho ao exercício e alguns parâmetros da função pulmonar como, capacidade funcional forçada e o VEF1 (GOMIDE et al., 2007).

\section{Pressão Expiratória Positiva (PEP)}

O sistema de pressão expiratória positiva é uma máscara facial e uma válvula unidirecional inspiratória, na qual possui uma resistência na fase expiratória de 10 a $20 \mathrm{cmH} 2 \mathrm{O}$. Essa resistência expiratória parece estabilizar as vias aéreas menores, assim precavendo seu colapso durante a tosse com expiração forçada. O paciente inspira e expira dentro da máscara em torno de 15 vezes e no período de descanso realiza-se respirações fora da máscara. Esse procedimento é repetido por volta de 20 minutos e comumente feito 2 vezes ao dia (MCLLWAINE et al., 2015; OLSÉN et al., 2015).

Esse recurso fisioterapêutico é indicado quando o objetivo do tratamento seja reduzir o aprisionamento de ar em indivíduos que apresentam asma e DPOC, mobilizar secreções em pacientes com fibrose cística e reverter atelectasias. E como resultado, aumento da expectoração de muco, da saturação de oxigênio e melhora na função pulmonar (SILVA \& BROMERSCHENCKEL, 2013).

\section{Pressão Expiratória Positiva Oscilatória - Flutter}

Flutter é um aparelho que combina as técnicas de Pressão Expiratória Positiva com oscilações de alta frequência, transportadas às vias aéreas. Esse aparelho é em formato de cachimbo que possui uma esfera de aço dentro e todas as vezes que o paciente expirar, o movimento da esfera irá criar uma certa pressão expiratória positiva e uma oscilação vibratória do ar dentro das vias aéreas. Ao utilizar esse método o paciente poderá controlar a pressão, modificando seus fluxos expiratórios e as oscilações serão alteradas pela alternância da inclinação do aparelho. O Flutter melhora os mecanismos de eliminação da secreção em pacientes fibrocísticos e é igualmente eficaz quando comparado a outras técnicas de desobstrução de vias aéreas (PRADO, 2011; DWYER et al., 2017). 


\section{Drenagem Autógena}

A drenagem autógena é uma técnica que utiliza o alto fluxo expiratório objetivando a acarretar oscilações brônquicas e, como resultado, mobilizar as secreções das vias aéreas mais distais para as centrais. Essa técnica é composta por 3 etapas: a primeira etapa consiste em "desprender" o muco periférico por meio de respirações a volume pulmonar baixo. A segunda etapa consiste em "coletar" a secreção por meio de respirações a médio volume. A última etapa consiste em "eliminar" o muco fazendo respirações com volume pulmonar alto (SILVA \& BROMERSCHENCKEL, 2013; WARNOCK \& GATES 2015). A drenagem autógena demonstrou resultados significativos na eficácia da expectoração da secreção. Dessa forma, ocorre o aumento do fluxo de ar nas vias aéreas, melhorando a curto prazo a capacidade vital forçada do paciente com fibrose cística (MORGAN et al., 2015).

\section{Fisioterapia nas alterações musculoesqueléticas}

O caráter progressivo da função pulmonar do indivíduo com fibrose cística reduz significativamente a capacidade de participação em atividades físicas. Os recorrentes processos inflamatórios do pulmão causam alterações metabólicas que levam à redução da densidade mineral óssea e da massa muscular. $O$ decrescimento da capacidade ventilatória coopera para a limitação física e aeróbica desses pacientes que relatam fadiga e dispneia após o exercício, afastando-os da prática de exercícios físicos. O sedentarismo e a inatividade para esses indivíduos causam uma diminuição progressiva do condicionamento físico, comprometimento da qualidade de vida e dispneia à pequenos esforços (SCHINDEL \& DONADIO, 2013; FRANCO et al., 2014; RADTKE et al., 2015).

A atividade física juntamente com o tratamento fisioterapêutico diário, deve ser sugerido e recomendado para os pacientes com fibrose cística para prevenir a piora da função pulmonar, estimular a desobstrução das vias aéreas, diminuir a incidência de infecção, melhorar a aptidão física e qualidade de vida. O treinamento frequente de exercício físico pode aumentar a capacidade anaeróbica e aeróbica e contribuir para o aumento da ventilação, ajudando na expectoração das secreções nas vias aéreas (WILLIAMS et al., 2010; ROVEDDER et al., 2014; BUTTON et al., 2016).

O tratamento fisioterapêutico não deve abordar somente a função respiratória, mas também na função muscular do paciente fibrocístico e incluir exercícios de cinesioterapia com resistência respeitando a tolerância máxima de cada indivíduo. A conciliação de treinamento de força muscular e treinamento aeróbico, aponta boa tolerância, mesmo para aqueles pacientes com volume expiratório no primeiro segundo menor do que 80\% (SCHINDEL \& DONADIO, 2013; SAVI et al., 2015)

A atividade física regular pode retardar o aparecimento da osteoporose, evitando uma diminuição na densidade mineral óssea. Outros ganhos com o treinamento físico é a maior sensação de bem-estar, redução da ansiedade e depressão e melhora da qualidade de vida, principalmente para pacientes com fibrose cística que tem como característica o déficit da capacidade respiratória cooperando para a limitação física (RADTKE et al., 2015). A fisioterapia respiratória associada com a atividade física aumenta o clearance das vias aéreas, melhora a capacidade vital, capacidade vital forçada e os sintomas em paciente com fibrose cística (CARDOSO \& VIANIA, 2011; SCHINDEL \& DONADIO, 2013). 


\section{Alterações no sistema digestivo}

As mutações no gene da cftr acarretam no mau funcionamento da proteína CFTR que irá afetar todas células secretoras do organismo. Devido a isso, o paciente apresenta alterações importantes no trato gastrintestinal e na função digestivo-absortiva. A proteína CFTR está posicionada na membrana apical dos enterócitos das células dos ductos biliares e pancreáticos, desempenhando um papel essencial na secreção de bicarbonato, cloro e fluidos. O funcionamento correto da proteína CFTR depende do transporte adequado de cloro e bicarbonato, havendo alteração de todo o processo digestivo e de absorção de nutrientes quando a secreção de íons está defeituosa (SILVA et al., 2015; BATTESTIN et al., 2016). As manifestações mais frequentes são a desnutrição calórica proteica, diarreia crônica, anemia, dor abdominal, constipação, edema, entre outros. Com a progressão da doença, o paciente apresenta tamanho reduzido, deformidades específicas e baixo peso, ocasionando a exclusão social do indivíduo, por conta própria ou por incapacidade (CONTO, 2014; REISINHO \& GOMES, 2016).

Além da insuficiência pancreática, outras manifestações digestivas são, o íleo meconial, síndrome da obstrução intestinal distal e a colonopatia fibrosante. O íleo meconial trata-se de uma obstrução pós-natal do trato intestinal inferior, logo após ao nascimento. Decorrente da impactação de mecônio dentro do íleo terminal, devido a alteração da motilidade do intestino e da secreção espessa. Sendo a manifestação mais precoce da fibrose cística e caracteristicamente, acontece em paciente que apresentam insuficiência pancreática (DEL CIAMPO et al., 2014; BOCZAR et al., 2015).

A síndrome de obstrução intestinal distal é semelhante ao íleo meconial, porém acomete mais em adolescentes e adultos. Igualmente, ocorre a impactação do mecônio no íleo terminal, cólon ascendente e ceco. Os fatores desencadeantes serão o uso de medicamentos que impedem a motilidade instestinal, a desidratação e o uso incorreto de reposição enzimática. A colonopatia fibrosante ocorre com maior frequência em crianças, que apresentam um quadro clínico inflamatório, fibrose progressiva e encurtamento da mucosa do cólon. Essa enfermidade está associada a utilização de altas doses de enzima pancreática e os principais sinais clínicos são, distensão abdominal, dificuldade para ganhar peso, hemorrogia digestiva, entre outros (NETO, 2009; COUTINHO et al., 2014).

Apesar da escassa literatura sobre o tratamento fisioterapêutico nessas alterações do sistema digestivo, alguns estudos sobre constipação intestinal e dor abdominal crônica relatam os benefícios das terapias manuais, atividades físicas e cinesioterapia. Esses recursos podem melhorar a dor, a qualidade vida e atenuar a incapacidade do paciente. Porém o tratamento deve ser individualizado, conforme as limitações do paciente. A terapia manual visceral é realizada na seguinte ordem: com a polpa digital, fazer uma pressão lenta e gradual, deslizando os dedos inicialmente na região do ceco, passando pelo cólon ascendente, cólon transverso, cólon descendente e finalizando em sigmoide. Essa técnica estimula e aumenta 0 peristaltismo, ajuda nas secreções glandulares, reduz o tempo de trânsito colônico e diminui a dor e o desconforto causado pelas disfunções digestivas (FERRAZ et al., 2013; BRAZ et al., 2013).

A atividade física regular, além de auxiliar na melhora da qualidade de vida e da capacidade funcional, acarreta mudanças hormonais e movimentos no intestino grosso que irão provocar efeitos mecânicos em todo o intestino, auxiliando o peristaltismo. Programas de cinesioterapia para o fortalecimento dos músculos 
abdominais e do assoalho pélvico melhoram também a motilidade gastrointestinal e ajudam na eliminação do bolo fecal (COX et al., 2015; GARCIA et al., 2016).

\section{Fisioterapia e a contribuição da família}

O paciente com fibrose cística necessita de uma abordagem individualizada, dando ênfase na gravidade da doença e nos aspectos clínicos, feita por uma equipe multidisciplinar. Por se tratar de uma doença sistêmica e com um tratamento complexo, o envolvimento e comprometimento da família são valiosos em todos os processos realizados, especialmente quando se trata de crianças e adolescentes. Muitas vezes, a equipe foca somente no tratamento da doença e nos aspectos do cuidar e esquece das necessidades pessoais do paciente e da família. Saber o que acontece no dia a dia do paciente com FC é imprescindível para que todos os profissionais envolvidos no tratamento desse indivíduo possam ter um olhar mais humanizado quanto ao processo saúde-doença e evolução (TAVARES et al., 2010; TAVARES et al., 2014).

A participação dos pais e/ou do cuidador nas sessões de fisioterapia torna-se fundamental para que o fisioterapeuta possa orientar e ensinar sobre os cuidados e exercícios a serem realizados diariamente em casa. E conscientizar sobre a importância da presença regularmente do paciente ao centro de reabilitação, para melhora da adesão e efetividade do programa de tratamento. Dessa forma, a cooperação dos familiares, paciente e/ou cuidador facilita para que o tratamento seja eficaz e que se estabeleça uma relação mútua de confiança, visando a melhora do prognóstico da doença (ALVES et al., 2007; RIZZO et al., 2015).

O paciente deve ser assíduo e colaborativo nas sessões de tratamento para tornar a fisioterapia mais efetiva. Adicionalmente, é importante destacar que a motivação é o segredo para a adesão ao tratamento, especialmente para os pacientes crônicos que são atendidos nos ambulatórios ou em domicílio pois, nenhum método ou tratamento será eficaz se for abandonado pelo paciente (PIZZIGNACCO et al., 2011; GOODFELLOW et al., 2015).

\section{CONSIDERAÇÕES FINAIS}

A partir da premissa de que a fisioterapia deve assistir ao paciente de forma holística, a presente revisão bibliográfica discorreu sobre técnicas para incrementar as funções respiratórias, musculoesquelética e gastrointestinal na fibrose cística. A compilação de técnicas incluiu também a abordagem de terapias manuais, que apesar de não promoverem a cura, contribuem para o melhor prognóstico, sobrevida e qualidade de vida dos pacientes com fibrose cística. $O$ tratamento fisioterapêutico proporciona a melhora global do quadro fisiopatológico dos pacientes com fibrose cística, em especial, com a participação da família e a motivação do paciente que contribuem valiosamente para o sucesso terapêutico.

\section{REFERÊNCIAS}

AGUIAR K. C. A.; NUCCI N. A. G.; HORTÊNCIO T. D. R.; RIBEIRO A. F.; RIBEIRO J. D. Viver com fibrose cística: a visão pessoal do adolescente brasileiro. Psicologia em estudo, v.21, n.2, p. 211-222, 2016. Disponível em: $<$ http://dx.doi.org/10.4025/psicolestud.v21i2.30494> 10.4025/psicolestud.v21i2.30494

AlVES C. A. D.; AGUIAR R. A.; ALVES A. C. S.; SANTANA M. S. Diabetes melito: 
uma importante co-morbidade da fibrose cística. Jornal Brasileiro de Pneumologia, v.33, n.2, p. 213-221, 2007. Disponível em: < http://dx.doi.org/10.1590/S1806-37132007000200017> doi: 10.1590/S180637132007000200017.

BATTESTIN B.; LIMA P. M.; FERREIRA S. H.; SILVA I. P.; PATROCÍNIO M. E.; PIERDONÁ N.; BANDEIRA N. F.; MONTE L. F. V. Relato de caso: Diagnóstico e manejo de paciente com Fibrose Cística em apresentação clássica. Revista de Medicina e Saúde de Brasília, v.5, n.1, p. 66-78, 2016. Disponível em: <https://portalrevistas.ucb.br/index.php/rmsbr/article/download/6752/4328>

BOCZAR M.; SAWICKA E.; ZYBERT K. Meconium ileus in newborns with cystic fibrosis - results of treatment in the group of patients operated on in the years 20002014. Developmental Period Medicine, v.XIX, n.1, p. 32-40, 2015. Disponível em: <http://psjd.icm.edu.pl/psjd/element/bwmeta1.element.psjd-ce76475b-11f7-4edb9460-9dea3292a4e6/c/2015-1-4.pdf>

BUTTON B. M.; WILSON C.; DENTICE R.; COX N. S.; MIDDLETON A.; TANNENBAUM E.; et al.,; Physioterapy for cystic fibrosis in Australia and New Zealand: a clinical practice guideline. Respirology, v.21, n.4, p. 656-667, 2016. Disponível em: <http://dx.doi.org/10.1111/resp.12764> doi: 10.1111/resp.12764

BRAZ M. M.; REAL A. A.; KELLING B. I.; STALLBAUM J. H.; POZZEBON N.M.; DIAS S. D.; BOCK T. H. O.; PIVETTA H. M. F. Efeitos da massagem sobre a constipação intestinal: uma revisão sistemática. Revista Biomotriz, v.7, n.1, p. 42-52, 2013. Disponível

em: <http://revistaeletronica.unicruz.edu.br/index.php/BIOMOTRIZ/article/download/165/p df $>$

CARDOSO R. M. T.; VIANA R. A. Intervenção da Fibrose Quística: uma revisão de literatura. Arquivo Médico, v.25, n.5-6, 2011. Disponível em: $<$ http://www.scielo.mec.pt/pdf/am/v25n5-6/v25n5-6a05.pdf>

CASTAÑOS C.; RENTERIA F. Consenso Nacional de Fibrosis Quística. Archivos Argentinos de Pediatría, 2008. Disponível em: <http://www.sap.org.ar/docs/profesionales/consensos/v106n5a12e.pdf>

CASTRO M. C. S.; FIRMIDA M. C. O tratamento na fibrose cística e suas complicações. Revista do Hospital Universitário Pedro Ernesto, UERJ, v.10, n.4, p. 82-108, 2011. Disponível em <http://revista.hupe.uerj.br/audiencia_pdf.asp?aid2=76\&nomeArquivo=v10n4a09.pd>

COHEN M. A.; RIBEIRO M. A. G. O.; RIBEIRO A. F.; RIBEIRO J. D.; MORCILLO A. M. Avaliação da Qualidade de vida de pacientes com fibrose cística por meio do Cystic Fibrosis Questionnaire. Jornal Brasileiro de Pneumologia, v.37, n.2, p. 184192, 2011. Disponível em: < http://dx.doi.org/10.1590/S1806-37132011000200008> doi: 10.1590/S1806-37132011000200008

CONTO C. L.; VIEIRA C. T.; FERNANDES K. N.; JORGE L. M.; CÂNDIDO G. S.; BARBOSA R. I.; DUTRA R. C. Prática fisioterapêutica no tratamento da fibrose cística. Arquivos Brasileiros de Ciências da Saúde, v.39, n.2, p. 96-100, 2014. 
Disponível em: < http://dx.doi.org/10.7322/abcshs.v39i2.629> doi: 10.7322/abcshs.v39i2.629

COUTINHO H. D. M.; FIGUEREDO F. G.; TINTINO S. R.; LIMA L. F.; FERREIRA J. V. A.; MARTINS G. M. A. B.; FREITAS M. A. Canais iônicos e Fibrose Cística. Revista Interfaces: Saúde, Humanas e Tecnologia, v.2, n.5, 2014. Disponível em: <http://dx.doi.org/10.16891/2317.434X.87> doi: 10.16891/2317.434X.87

COUTINHO C. A. A. C.; MARSON F. A. L.; RIBEIRO A. F.; RIBEIRO J. D.; BERTUZZO C. S. Mutações do gene cystic fibrosis transmembrane conductance regulator em um centro de referência para fibrose cística. Jornal Brasileiro de Pneumologia, v.39, n.5, p. 555-561, 2013. Disponível em: <http://dx.doi.org/10.1590/S1806-37132013000500005> doi: 10.1590/S180637132013000500005

COX N. S.; ALISSON J. A.; BUTTON B. M.; WILSON J. W.; MORTON J. M.; HOLLAND A. E. Physical activity participation by adults with cystic fibrosis: an observation study. Respirology, v.21, n.3, p. 511-518, 2016. Disponível em: < http://dx.doi.org/ 10.1111/resp.12719 doi: 10.1111/resp.12719

DALCIN P. T. R.; SILVA F. A. A. Fibrose Cística no adulto: aspectos diagnósticos e terapêuticos. Jornal Brasileiro de Pneumologia, v.34, n.2, p. 107-117, 2008. Disponível em < http://www.scielo.br/pdf/jbpneu/v34n2/v34n2a08.pdf>

DEL CIAMPO I. R. L.; OLIVEIRA T. Q.; CIAMPO L. A.; SAWAMURA R.; TORRES L. A. G. M. M.; AUGUSTIN A. E.; FERNANDES M. I. M. Early manifestations of cystic fibrosis in a premature patient with complex meconium ileu at birth. Revista Paulista de Pediatria, v.33, n.2, p. 241-245, 2014. Disponível em: <http://dx.doi.org/ 10.1016/j.rpped.2014.12.004> doi: 10.1016/j.rpped.2014.12.004

DONADIO M. V. F.; SOUZA G. C.; TIECHER G.; HEINZMANN-FILHO J. P.; PAIM T. F.; HOMMERDING P. X.; MAROSTICA P. J. C. Bone Mineral density, pulmonary function, chronological age, and age at diagnosis in children and adolescents with cystic fibrosis. Jornal de Pediatria, v.89, n.2, p. 151-157, 2013. Disponível em: <http://dx.doi.org/10.1016/j.jped.2013.03.008> doi: 10.1016/j.jped.2013.03.008

DWYER T. J.; ZAINULDIN R.; DAVISKAS E.; BYE P. T. P.; ALISSON J. A. Effects of treadmill exercise versus Flutter on repiratory flow and sputum properties in adults with cystic fibrosis: a randomize, controlled, cross-over trial. BMC Pulmonary Medicine, v.17, n.14, p. 2-8, 2017. Disponível em: <http://dx.doi.org/10.1186/s12890016-0360-8> doi: 10.1186/s12890-016-0360-8

FEITEN T. S.; FLORES J. S.; FARIAS B. L.; ROVEDDER P. M. E.; CAMARGO E. G.; DALCIN P. T. R.; ZIEGLER B. Fisioterapia Respiratória: um problema de crianças e adolescente com fibrose cística. Jornal Brasileiro de Pneumologia, v.42, n.1, p. 29-34, 2016. Disponível em: <http://dx.doi.org/10.1590/S1806-37562016000000068> doi: 10.1590/S1806-37562016000000068

FERRAZ B. B.; MARTINS M. R. I.; FOSS M. H. D. A. Impact of manual visceral therapy to improve the quality of life of chronic abdominal pain patients. Revista Dor, 
v.14, n.2, p. 124-128, 2013. Disponível em: <http://dx.doi.org/10.1590/S180600132013000200010 > doi: 10.1590/S1806-00132013000200010

FLUME P. A.; ROBINSON K. A.; O' SULLIVAN B. P.; FINDER J. D.; VENDER R. L.; WILLEY-COURAND D. B.; WHITE T. B.; MARSHALL B. C. Cystic Fibrosis Pulmonary Guidelines: Airway Clearance Therapies. Respiratory Care, v.54, n.4, p. 522-537, 2009. Disponível em: < http://rc.rcjournal.com/content/54/4/522.full.pdf>

FRANCO C. B.; RIBEIRO A. F.; MORCILLO A. M.; ZAMBON M. P.; ALMEIDA M. B.; ROZOV T. Efeitos do método Pilates na força muscular e na função pulmonar de pacientes com fibrose cística. Jornal Brasileiro de Pneumologia, v.40, n.5, p. 521 527, 2014. Disponível em: <http://dx.doi.org/10.1590/S1806-37132014000500008> doi: 10.1590/S1806-37132014000500008

GARCIA L. B.; BERTOLINI S. M. M. G.; SOUZA M. V.; SANTO M. S. F.; PEREIRA C. O. M. Constipação intestinal: aspectos epidemiológicos e clínicos. Revista Saúde e Pesquisa, v.9, n.1, p. 153-162, 2016. Disponível em: <http://dx.doi.org/10.17765/19831870.2016v9n1p153-162> doi: 10.17765/1983-1870.2016v9n1p153-162

GOODFELLOW N. A.; HAWWA A. F.; REID A. J.; HOME R.; SHIELDS M. D.; MCELNAY J. C.; Adherence to treatment in children and adolescents with cystic fibrosis: a cross-sectional, multi-method study investigating the influence of beliefs about treatment and parental depressive symptoms. BMC Pulmonary Medicine, v.15, n.43, p. 1-10, 2015. Disponível em: <http://dx.doi.org/10.1186/s12890-0150038-7> doi: 10.1186/s12890-015-0038-7

GOMIDE L. B.; SILVA C. S.; MATHEUS J. P. C.; TORRES L. A. G. M. M. Atuação da fisioterapia respiratória em pacientes com fibrose cística: uma revisão de literatura. Arquivo de Ciências da Saúde, v.14, n.4, p. 227-233, 2007. Disponível em: <http://www.habdirect.co.uk/media/wysiwyg/pdfs/Shaker-

etc/GOMIDE_RespiratoryPhysiotherapyInPatientsWithCysticFibrosisAliteratureRevie w.pdf>

HEINZMANN-FILHO J. P.; PINTO L. A.; MAROSTICA P. J. C.; DONADIO M. V. F. Variação na função pulmonar está associada com os piores desfechos clínicos em indivíduos com fibrose cística. Jornal Brasileiro de Pneumologia, v.41, n.6, p. 509515, 2015. Disponível em: <http://dx.doi.org/10.1590/S1806-37562015000000006 > doi: 10.1590/S1806-37562015000000006

IKE D.; LORENZO V. A. P. D.; COSTA D. JAMAMI M. Drenagem postural: prática e evidência. Fisioterapia Movimento, Curitiba, v.22, n.1, p. 11-17, 2009. Disponível em: < http://www2.pucpr.br/reol/index.php/rfm?dd99=pdf\&dd1=2607>

LIEBANO R. E.; HASSEN A. M. S.; RACY H. H. M. J.; CORRÊA J. B. Principais manobras cinesioterapêuticas manuais utilizadas na fisioterapia respiratória: descrição das técnicas. Revista Ciência Médica, v.18, n.1, p. 35-45, 2009. Disponível em: < $\quad$ http://periodicos.puccampinas.edu.br/seer/index.php/cienciasmedicas/article/download/652/632>

MORGAN K.; OSTERLING K.; GILBERT R.; DECHMAN G. Effects of autogenic ENCICLOPÉDIA BIOSFERA, Centro Científico Conhecer - Goiânia, v.14 n.25; p. 1486 2017 
drainage on sputum recovery and pulmonary function in people with cystic fibrosis: a systematic review. Physioterapy Canada, v.67, n.4, p. 319-326, 2015. Disponível em: <http://dx.doi.org/10.3138/ptc.2014-64> doi: 10.3138/ptc.2014-64

MCLLWAINE M.; BUTTON B.; DAWN K. Positive expiratory pressure physiotherapy for airway clearance in people with cystic fibrosis. Cochrane Database, v.17, n.6, 2015. Disponível em: <http://dx.doi.org/10.1002/14651858.CD003147.pub4> doi: 10.1002/14651858.CD003147.pub4

NOBERTO NETO L. Fibrose Cística Enfoque Multidisciplinar. Secretaria de Estado da Saúde, Florianópolis, 2ํe edição revisada e ampliada, 688 p, 2009.

OLIVEIRA E. A. R.; GOMES E. L. F. D. Evidência científica das técnicas atuais e convencionas de fisioterapia respiratória em pediatria. Fisioterapia Brasil, v.17, n.1, p. 88-97, 2016. Disponível em: <http://www.portalatlanticaeditora.com.br/index.php/fisioterapiabrasil/article/download /30/320>

OLSÉN M. F.; LANNEFORS L.; WESTERDAHL E. Positive expiratory pessure Common clinical applications and physiological effects. Respitory Medicine, v.109, n.13, p. 297-307, 2015. 2 Disponível em: <http://dx.doi.org/10.1016/j.rmed.2014.11.003> doi: 10.1016/j.rmed.2014.11.003

PIZZIGNACCO T. P.; MELLO D. F.; LIMA R. G. A experiência da doença na fibrose cística: caminhos para cuidado integral. Revista da Escola de Enfermagem, v.45, n.3, p. 638-644, 2011. Disponível em: <http://dx.doi.org/10.1590/S008062342011000300013> doi: 10.1590/S0080-62342011000300013

PRADO S. T. O papel da fisioterapia na fibrose cística. Revista do Hospital Universitário Pedro Ernesto, v.10, n.4, p. 118-125, 2011. Disponível em: < http://revista.hupe.uerj.br/audiencia_pdf.asp?aid2=78\&nomeArquivo=v10n4a11.pdf>

QUON B. S.; ROWE S. M. New and emerging targeted therapies for cystic fibrosis. The BMJ Publishing Group Limited, 2016. Disponível em: <http://dx.doi.org/10.1136/bmj.i859> doi:10.1136/bmj.i859

RADTKE T.; NOLAN S. J.; HEBESTREIT H.; KRIEMLER S. Physical exercise training for cystic fibrosis. Cochrane Database, v.28, n.6, 2015. Disponível em: <http://dx.doi.org/10.1002/14651858.CD002768.pub3> 10.1002/14651858.CD002768.pub3

REISINHO M. C.; GOMES B. O adolescente com fibrose cística: crescer na diferença. Revista Portuguesa de Enfermagem de Saúde Mental, especial 3, p. 85-94, 2016. Disponível em <http://dx.doi.org/10.19131/rpesm.0123> doi: 10.19131/rpesm.0123

RIZZO L. C.; FISCHER G. B.; MARÓSTICA P. J. C.; MOCELIN H. T. Profile of cystic fibrosis in two reference center in Southern Brazil. Revista da Associação Médica Brasileira, v.61, n.2, p. 150-155, 2015. Disponível em <http://dx.doi.org/10.1590/1806-9282.61.02.150> doi: 10.1590/1806-9282.61.02.150 
ROVEDDER P. M. E.; FLORES J.; ZIEGLER B.; CASAROTTO F.; JAQUES P.; BARRETO S. S. M.; DALCIN P. T. R. Exercise programme in patients with cystic fibrosis: a randomized controlled trail. Respiratory Medicine, v.108, n.8, p. 11341140, 2014. Disponível em: < http://dx.doi.org/10.1016/j.rmed.2014.04.022> doi: 10.1016/j.rmed.2014.04.022

SAVI D.; DI PAOLO M.; SIMMONDS N.; ONORATI P.; INTERNULLO M.; QUATTRUCCI S.; WINSTON B.; LAVENEZIANA P.; PALANGE P.; Relationship between daily physical activity and aerobic fitness in adults with cystic fibrosis. BMC Pulmonary Medicine, v.15, n.59, 2015. Disponível em: < http://dx.doi.org/10.1186/s12890-015-0036-9> doi: 10.1186/s12890-015-0036-9

SILVA FILHO L. V. R. F.; FERREIRA F. A. REIS F. J. C.; BRITO M. C. A.; LEVY C. E.; CLARK O.; RIBEIRO J. D.; Infecção por Pseudomonas aeruginosa em pacientes com fibrose cística: evidências científicas sobre o impacto clínico, diagnóstico e tratamento. Jornal Brasileiro de Pneumologia, v.39, n.4, p. 495-512, 2013. Disponível em: < http://dx.doi.org/10.1590/S1806-37132013000400015> doi: 10.1590/S1806-37132013000400015

SILVA K. M.; BROMERSCHENCKEL A. I. M.; Fisioterapia respiratória nas doenças pulmonares obstrutivas crônicas. Revista HUPE, v.12, n.2, p. 94-100, 2013. Disponível em:

http://revista.hupe.uerj.br/audiencia_pdf.asp?aid2=398\&nomeArquivo=v12n2a12.pd>

SILVA L. M.; MONTEIRO E. S.; MIRANDA B. S.; SILVA C. R. L.; COSTA A. S. M. Influence of method Thoracic-Abdominal Rebalance on parameters cardiorespiratory in patients with cystic fibrosis. Manual Therapy, Posturology \& Rehabilitation Journal, v.13, n.3, p. 1-16, 2015. Disponível em: <http://dx.doi.org/10.17784/mtprehabjournal.2015.13.233> doi: 10.17784/mtprehabjournal.2015.13.233

SILVA M. A.; PFEIFER L. I.; Reabilitação pulmonar de crianças com fibrose cística do Estado do Pará. Fisioterapia em Movimento, v.20, n.4, p. 73-81, 2007. Disponível em: < http://www2.pucpr.br/reol/pb/index.php/rfm?dd1=1778\&dd99=view\&dd98=pb>

SUNDELL M. O. T.; MENESES D. G.; RIBEIRO A. F.; PINTO E. A. L. C.; HESSEL G. Kwashiorkor e distúrbio de coagulação - apresentação atípica de fibrose cística. Revista Paulista de Pediatria, v.30, n.1, p. 139-143, 2012. Disponível em: < http://dx.doi.org/10.1590/S0103-05822012000100020> doi: 10.1590/S010305822012000100020

SCHINDEL C. S.; DONADIO M. V. F.; Efeitos de programas de exercício físico em pacientes com fibrose cística. Scientia Medica, v.23, n.3, p. 187-190, 2013. Disponível em:

https://www.researchgate.net/profile/Marcio_Donadio/publication/286066735_Effects _of_physical_exercise_programs_in_patients_with_cystic_fibrosis/links/56e2fbb908a e1c52fafdacōa.pdf>

TAVARES K. O.; CARVALHO M. D. B.; PELLOSO S. M. O que é ser mãe de uma criança com fibrose cística. Revista Gaúcha Enfermagem, v.31, n.4, p. 723-729, 
2010. Disponível em: < http://dx.doi.org/10.1590/S1983-14472010000400016> doi: $10.1590 /$ S1983-14472010000400016

TAVARES K. O.; CARVALHO M. D. B.; PELLOSO S. M. Dificuldades vivenciadas por mães de pessoas com fibrose cística. Texto Contexto Enfermagem, v.23, n.2, p. 294-300, 2014. Disponível em: <http://dx.doi.org/10.1590/010407072014000050013> doi: 10.1590/0104-07072014000050013

TORRES L.; HERNANDEZ J. L. J.; ALMEIDA G. B.; GOMIDE L. B.; AMBRÓSIO V.; FERNANDES M. I. M. Avaliação clínica, nutricional e espirométrica de pacientes com fibrose cística após implantação de atendimento multidisciplinar. Jornal Brasileiro de Pneumologia, v.36, n.6, p. 731-737, 2010. Disponível em: <http://dx.doi.org/10.1590/S1806-37132010000600010> doi: 10.1590/S180637132010000600010

WARNOCK L.; GATES A.; Chest physiotherapy compared to no chest physiotherapy for cystic fibrosis. Cochrane Database, v.4, n.9, 2015. Disponível em: <http://dx.doi.org/10.1002/14651858.CD001401.pub2> doi: 10.1002/14651858.CD001401.pub2

WILLIANS C. A.; BENDEN C.; STEVENS D.; RADTKE T.; Exercise training in children and adolescents with cystic fibrosis: theory into practice. International Journal of Pediatrics, p. 1-7 2010. Disponível em: <http://dx.doi.org/10.1155/2010/670640> doi: http://dx.doi.org/10.1155/2010/670640

ZIEGLER B.; ROVEDDER P. M. E.; DALCIN P. T. R; MENNA-BARRETO S. S. Padrões ventilatórios na espirometria em pacientes adolescentes e adultos com fibrose cística. Jornal Brasileiro de Pneumologia, v.35, n.9, p. 854-859, 2009. Disponível em: <http://dx.doi.org/10.1590/S1806-37132009000900006> doi: $10.1590 /$ S1806-37132009000900006 